Review article

\title{
Bone augmentation using autogenous bone versus biomaterial in the posterior region of atrophic mandibles: A systematic review and meta- analysis
}

\section{Cecília Alves de Sousa ${ }^{\mathrm{a}, *}$, Cleidiel Aparecido Araújo Lemos ${ }^{\mathrm{a}}$, Joel Ferreira Santiago-Júnior ${ }^{\mathrm{b}}$, Leonardo Perez Faverani ${ }^{\mathrm{a}}$, Eduardo Piza Pellizzer ${ }^{\mathrm{a}}$}

${ }^{a}$ Aracatuba Dental School, São Paulo State University, São Paulo, Brazil

${ }^{\mathrm{b}}$ University of Sacred Heart, São Paulo, Brazil

\section{A R T I C L E I N F O}

\section{Keywords:}

Bone grafting

Mandible

Dental implants

\begin{abstract}
A B S T R A C T
Objectives: This systematic review and meta-analysis aimed to answer the PICO question: "Do patients who have received bone grafts with bone substitute (biomaterials) present bone gain (before implant installation), complications, and implant survival rates similar to autogenous grafts when used in the posterior mandible region?". Data: This review followed the PRISMA statement and has been registered at PROSPERO (CRD42016048471). Studies published in English, randomized controlled and/or prospective clinical trials with at least 10 patients, and studies that compared grafts with bone substitutes to autogenous bone grafts (split-mouth design) were included.

Sources: An electronic search and a manual search were conducted in PubMed/MEDLINE, Scopus, and Cochrane databases up to April 2018.

Study selection: Our initial search yielded 640 articles; we selected four articles that met the inclusion criteria. All selected studies used a split-mouth design.

Results: Our analysis revealed no significant difference between the biomaterial and autogenous groups in terms of bone gain ( $\mathrm{P}=0.11$; mean difference $[\mathrm{MD}]$ : 0.59 ; 95\% confidence interval [CI]: $-0.13-1.31$ ) or complication rate ( $\mathrm{P}=0.72$; risk ratio [RR]: 1.25 ; 95\% CI: 0.37-4.23). Sixty-six implants were installed in the biomaterial group and 63 in the autogenous group; these showed no significant difference in implant survival rate $(\mathrm{P}=0.50$; RR: 1.57 ; 95\% CI: 0.43-5.81).

Conclusion: We conclude that biomaterials or autogenous bone are indicated for the reconstruction of the posterior mandibular atrophic region, without lowering implant survival.
\end{abstract}

\section{Introduction}

Resorption of the maxillary and mandibular bones is a physiological event that occurs over time after tooth loss, and leads to a state of partial or total edentulous alveolar ridge atrophy [1]. Oral rehabilitation through oral implants is a suitable method to restore oral aesthetics and function with predictable results [2,3]. However, a prerequisite for obtaining a successful outcome with implants is minimum bone width and height of the receiving site, which allows for implant installation in the appropriate place and ensures a functional and aesthetic restoration [4-6].

In cases of alveolar ridges with insufficient bone height and volume, additional surgical procedures for reconstruction and enlargement of the deficient regions are needed. Several techniques have been developed to reconstruct deficient mandibular alveolar ridges for implant placement. These include a one-stage simultaneous approach and a two-stage approach [6,7]. These procedures involve the use of bone grafts composed of different types of materials (e.g., autogenous, xenogenous, or other bone substitutes) and can be executed by guided bone regeneration alone or in combination with graft procedures.

Autogenous bone is considered the gold standard in graft surgeries because of its biocompatibility and its osteoinductive, osteoconductive, and osteogenic properties [8,9]. However, the limited availability of intraoral donor sites and the high morbidity associated with the use of extraoral donor sites have made the use of autogenous bone for rehabilitation difficult $[10,11]$. In view of these difficulties, several

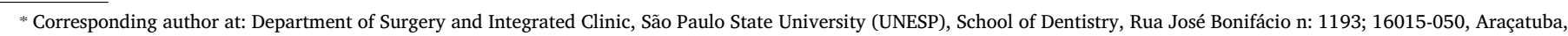
São Paulo, Brazil.

E-mail address: ceciliasousa_alves@hotmail.com (C.A. de Sousa). 
materials have been used to replace autogenous bone, especially bovine organic bone, which has fundamental characteristics of biocompatibility and osteoconductivity and provides an ideal framework for new bone formation $[12,13]$. Bovine organic bone is being widely used for vertical/horizontal bone augmentation $[14,15]$ and maxillary sinus lift [16].

Several techniques for bone defect reconstruction in the posterior mandibular region have been developed to achieve adequate bone volume for implant installation $[17,18]$. This region is considered critical because it is in close contact with the inferior alveolar nerve and is subject to rapid bone resorption with aging, especially after the loss of dental elements [17,19]. In addition, biological factors such as bone width of the recipient area and amount of bone wall affect bone graft stability. These characteristics may affect blood supply during bone graft repair, thereby affecting its ability to heal. This may also lead to greater bone resorption of the grafted bone or even graft loss. Therefore, the posterior mandibular region is an extremely critical region for bone augmentation procedures in oral rehabilitation [17].

The purpose of this systematic review and meta-analysis was to evaluate and compare the clinical outcomes of bone augmentation using autogenous bone and biomaterial in the posterior mandibular atrophic region prior to implant installation. In addition, this study evaluated the survival of implants installed in these grafted regions on the basis of the following hypotheses: 1) There is no difference between the use of biomaterials and autogenous bone graft with respect to bone gain. 2) The complication and survival rates of the implants are not influenced by the type of bone graft.

\section{Materials and methods}

\subsection{Protocol and registration}

This systematic review was based on the Preferred Reporting Items for Systematic Reviews and Meta-Analyses (PRISMA) checklist structure [20] and was conducted in accordance with models proposed in published reports $[6,7,21]$. Moreover, this study was registered on the international prospective register of systematic reviews (PROSPERO: CRD42016048471).

\subsection{Eligibility criteria}

The studies selected for this analysis followed the criteria established by the PICO index, defining the following question: "Do bone grafts with bone substitute (biomaterials) present bone gain similar to autogenous grafts when used in the posterior mandible region?". Studies included patients requiring a bone graft in the posterior mandibular region for implant installation. Patients who received grafts with bone substitutes were compared with those who received autogenous bone grafts in the posterior mandibular region for implant installation, with respect to the following outcomes: bone gain before implant installation (primary outcome), the complication rates, and survival of implants installed in the grafted region (secondary outcomes).

The inclusion criteria were as follows: studies published in the English language, randomized controlled and/or prospective clinical trials with at least 10 patients, and studies that compared patients who received grafts with bone substitutes with those who received autogenous bone grafts (split-mouth design). The exclusion criteria were as follows: animal studies and in vitro studies, studies with patients who underwent bone graft surgery without the use of autogenous bone and/ or biomaterials, and studies with patients who underwent graft surgery in the maxillary region.

\subsection{Information sources and search strategy}

The search for the studies was independently performed by two previously calibrated reviewers (C.A.S. and C.A.A.L.). The authors conducted an electronic search of the PubMed/MEDLINE, Scopus, and Cochrane databases for articles published up to April 2018. All studies identified by the inclusion criteria were analyzed, and the corresponding authors of these studies were contacted to identify possible additional information. The search was performed using the following search terms: "bone graft AND vertical bone augmentation OR bone graft AND posterior mandible." The search strategy was as follows: ("bone transplantation"[MeSH Terms] OR ("bone"[All Fields] AND "transplantation"[All Fields]) OR "bone transplantation"[All Fields] OR ("bone"[All Fields] AND "graft"[All Fields]) OR "bone graft"[All Fields]) AND (vertical[All Fields] AND ("bone and bones"[MeSH Terms] OR ("bone"[All Fields] AND "bones"[All Fields]) OR "bone and bones"[All Fields] OR "bone"[All Fields]) AND augmentation[All Fields]) OR ("bone transplantation"[MeSH Terms] OR ("bone"[All Fields] AND "transplantation"[All Fields]) OR "bone transplantation"[All Fields] OR ("bone"[All Fields] AND "graft"[All Fields]) OR "bone graft"[All Fields]) AND (posterior[All Fields] AND ("mandible"[MeSH Terms] OR "mandible"[All Fields])).

The same researchers also manually searched for articles published in the journals Clinical Implant Dentistry and Related Research, Clinical Oral Implant Research, Implant Dentistry, International Journal of Oral and Maxillofacial Surgery, Journal of Clinical Periodontology, Journal of Dental Research, Journal of Dentistry, Journal of Maxillofacial and Oral Surgery, Journal of Oral Implantology, Journal of Periodontology, and International Journal of Oral and Maxillofacial Implants. All discrepancies related to the search in the databases and manual searching were analyzed and resolved by the third reviewer (J.F.S.J.) in a consensus meeting.

\subsection{Data collection process}

One of the authors (C.A.S.) collected relevant information from the articles and a second author (L.P.F.) reviewed all the collected information. The variables collected from the articles were as follows: author/year, type of study, number of patients, number of implants, characteristics of the implants, mean age, graft donor site, biomaterials, stabilizations of the bone graft, complications of the graft, number of implants survived, and bone gain.

\subsection{Risk of bias}

The risk of bias in the studies included was assessed independently by two authors (C.A.S. and C.A.A.L.). The Cochrane Collaboration's tool for assessing the risk of bias was used to assess the quality of the studies included in this review. This tool addressed six specific domains, namely, random sequence generation, allocation concealment, blind outcome assessment, incomplete outcome data, selective outcome reporting, and other biases. The classification was based on judgment related to the risk of bias and was defined as low, unclear, or high risk.

\subsection{Summary measures}

The influence of different bone grafts on bone gain was evaluated on the basis of a continuous outcome through the mean difference, while the survival rate of the implants was evaluated using a dichotomous outcome through the risk ratio (RR), both with a $95 \%$ confidence interval (CI). Analyses were performed using the software program Review Manager 5.3 (The Cochrane Library). P values $<0.05$ were considered to indicate statistical significance.

The fixed-effects model was used in situations with a low heterogeneity index, and the random-effects model was utilized in situations with a high heterogeneity index between the trials. The heterogeneity was evaluated using the $\mathrm{Q}\left(\mathrm{x}^{2}\right)$ method and the $I^{2}$ value was measured. The statistical value of $I^{2}$ was used to analyze variations in heterogeneity, and $I^{2}>75 \%$ (range, $0-100 \%$ ) was considered to indicate relevant heterogeneity $[22,23]$. 
Table 1

Reasons for exclusion of studies.

\begin{tabular}{|c|c|}
\hline Studies excluded & Justifications for exclusion \\
\hline Amorfini et al., 2014 & Evaluation of allograft with association with growth factors \\
\hline Brandtner et al., 2014 & Retrospective study \\
\hline Cha et al., 2014 & Retrospective study with evaluation of maxilla and mandible, without separation of the results between one region and another \\
\hline Checchi et al., 2018 & Case Series (less than 10 patients) \\
\hline Esposito et al., 2014 & Bio-Oss in block X particulate, without use of autogenous graft bone. Evaluation of a short implant installation in the grafted region \\
\hline Felice et al., 2008 & Less follow-up than the study by the same author in $2009^{\circ}$ \\
\hline Hu et al., 2018 & Case Series (less than 10 patients) \\
\hline Laino et al., 2014 & Histological evaluation of allograft $\mathrm{X}$ biomaterial \\
\hline Lizio et al., 2014 & Evaluation of alveolar reconstruction with titanium meshes \\
\hline Misch et al., 2015 & Does not use autogenous graft \\
\hline Spinato et al., 2012 & Immediate installation of implants comparing grafted area $\mathrm{X}$ without graft \\
\hline Voss et al., 2016 & Retrospective study \\
\hline Leong et al., 2015 & Graft with particulate X-block biomaterial, without autogenous graft evaluation \\
\hline Lopez-Cédrum et al., 2011 & A retrospective study evaluating the sandwich technique with only autogenous bone \\
\hline Martinez et al., 1999 & Case series \\
\hline Nissan et al., 2011 & Allograft analysis only \\
\hline Novel et al., 2012 & Retrospective study of allograft \\
\hline Palarie et al., 2011 & Effect of surface treatment of implants \\
\hline Pikos et al., 2003 & Literature review \\
\hline Rocchietta et al., 2016 & Only autogenous graft evaluation: particulate $\mathrm{X}$ block \\
\hline Ronda et al., 2014 & Evaluation of two membranes: d-PTFE X e-PTFE \\
\hline
\end{tabular}

\subsection{Additional analysis}

The kappa coefficient value was calculated to determine interreader agreement in the study, to evaluate the titles and abstracts selected, and to obtain a test of agreement (twice) for the databases PubMed/MEDLINE ( $\kappa=0.71$ and $0.48 ; 1)$, Cochrane $(\kappa=1 ; 1)$, and Scopus $(\kappa=1 ; 1)$.

\section{Results}

\subsection{Study selection}

A search of the databases retrieved 744 references, including 322 from PubMed/MEDLINE, 334 from Scopus, and 88 from the Cochrane database. Twenty-five studies met the inclusion/exclusion criteria applied to the titles and abstracts. Twenty-one of these studies were excluded because they did not meet the inclusion criteria after the reviewers read the entire articles (Table 1). Finally, four studies were selected for qualitative and quantitative analyses (Fig. 1).

\subsection{Characteristics of the studies}

Among the four selected studies, only one was classified as prospective; the other studies were randomized controlled trials. All studies were published in the period 2009-2015. Among all the patients studied, only one patient was lost to follow-up [24]. The mean age of the patients was 53.66 years.

All the included studies used a split-mouth design (one side: biomaterial; other side: autogenous bone). Only one of the studies used a graft with autogenous iliac crest bone, requiring surgery in the hospital under general anesthesia [25]. The other studies obtained grafts from intraoral sites, such as the oblique line. The mean follow-up period of the bone grafts in the evaluated studies, prior to implant placement, was 5.5 months (range: 4-6 months). Two studies performed histological analysis of the grafts [24,26]. Sixty-three implants were installed in the autogenous group and 66 in the biomaterial group. The mean follow-up period was 7.6 months, adding up to an average follow-up period of 13.1 months from the beginning of the surgical treatment for bone augmentation until the period of osseointegration of the implants (Table 2).

\subsection{Bone gain (before implant installation)}

All included studies evaluated bone gain [24-27]. A specific analysis comparing patients who received autogenous grafts and those who received biomaterial grafts revealed no significant difference between biomaterials and autogenous bone grafts in terms of bone gain ( $\mathrm{P}=0.11$; mean difference [MD]: 0.59; 95\% CI: -0.13-1.31; Fig. 2).

\subsection{Complication rate}

Two studies reported complication rates [25,27]. In an analysis assessing the reported complications in relation to the different types of bone grafting, a significant difference between the biomaterial and autogenous groups was not identified $(\mathrm{P}=0.72$; risk ratio $[\mathrm{RR}]$ : 1.25 ; 95\% CI: 0.37-4.23; Fig. 3).

\subsection{Implant survival rate}

Three studies reported data about the implant survival rate $[24,25,27]$. In an analysis reviewing the implants installed after the grafting surgery, 66 implants installed in the biomaterial group and 66 in the autogenous group were identified. No significant difference was observed between the graft types with respect to the survival rate of the implants ( $\mathrm{P}=0.50$; RR: 1.57; 95\% CI: 0.43-5.81; Fig. 4).

\subsection{Risk of bias in studies}

The heterogeneity of the studies was considered low with respect to bone gain $\left(\chi^{2}=0.57 ; \mathrm{P}=0.90 ; I^{2}=0 \%\right)$, bone graft complications ( $\chi^{2}$ $\left.=2.79 ; \mathrm{P}=0.09 ; I^{2}=64 \%\right)$, and implant survival rate $\left(\chi^{2}=2.06\right.$; $\mathrm{P}=0.36 ; I^{2}=3 \%$ ). Therefore, the fixed-effects model (IV, 95\% CI) was employed. The funnel graph showed evident symmetry in the distribution of the studies, indicating there was adequate homogeneity in the analyses of bone gain, complications in bone grafting, and implant survival rate (Fig. 5).

The Cochrane criteria indicated a low risk of bias for the included studies. One prospective study did not perform randomization and allocation of patients [24]. The blinding of patients or surgeons was unclear in two of the selected studies [24,27]. All studies were found adequate with a low risk of bias for the blinding of outcome assessment and incomplete outcome data. They were also free of selective outcome reporting and other sources of bias (Fig. 6). 


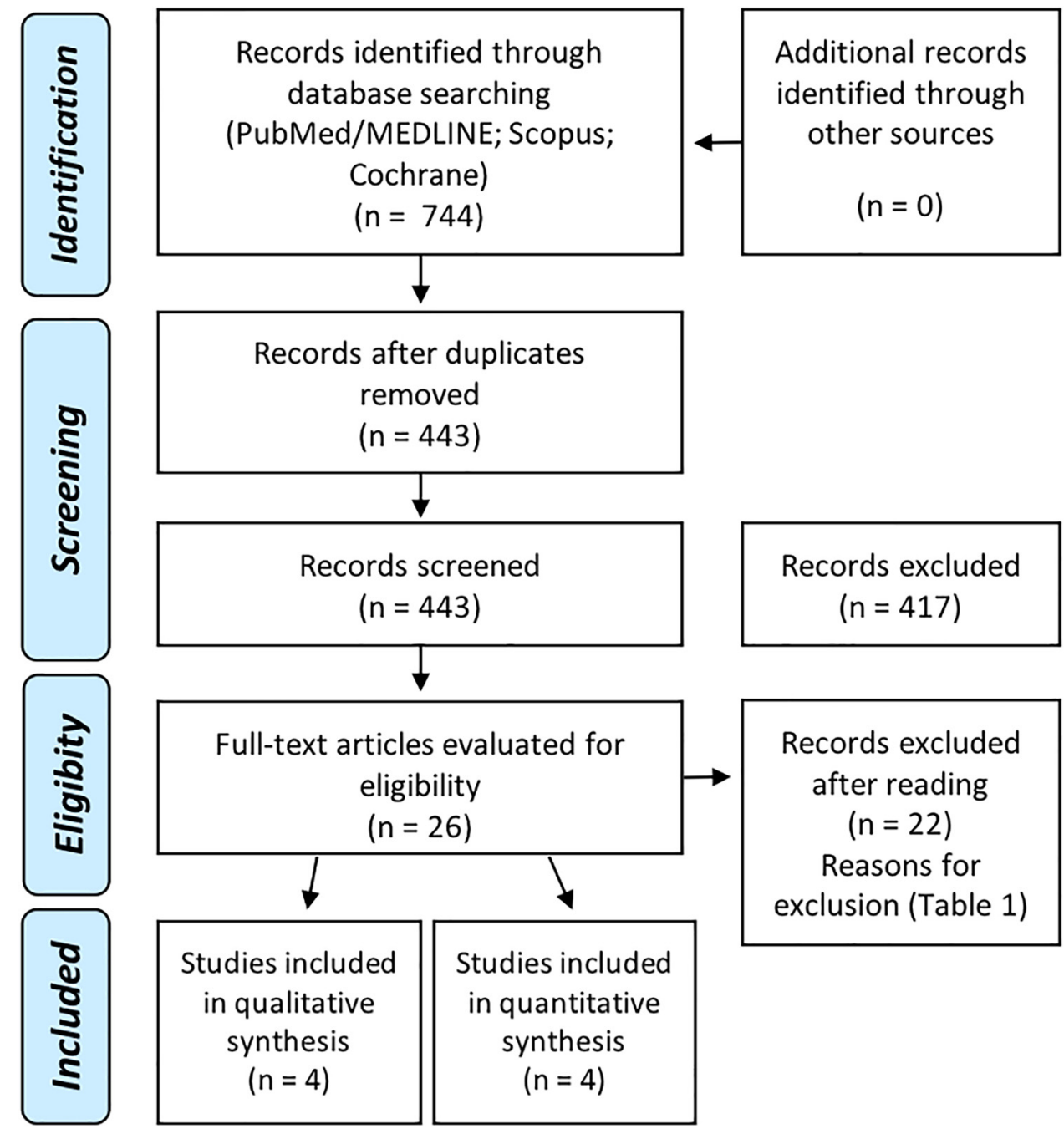

Fig. 1. Flowchart describing the search strategy.

\section{Discussion}

The investigation of vertical bone gain in the posterior mandibular atrophic region through this systematic review showed that the selected articles had a high level of evidence, since four of these were categorized as randomized controlled trials and one as a prospective study. In addition, all studies presented a comparison of both techniques of bone reconstruction in the same patient (split-mouth design), which reinforced the methodological quality and reliability of the results analyzed.

The meta-analysis revealed similarity for bone gain parameters regardless of the grafting material used, biomaterials (Bio-Oss and Osteogen) or autogenous bone, for reconstruction in the posterior mandibular atrophic region; therefore, the first hypothesis was accepted. However, the biomaterials group showed a favorable trend toward displacement (as shown by the diamond) in the forest-plot graph (Fig. 2).

These findings are corroborated by histological biopsy results $[25,26]$, which showed higher bone turnover for the autogenous grafts. This may justify the more favorable trend for vertical bone gain with the use of biomaterials than with the use of autogenous bone, which are fundamentally osteoinductive. Felice et al. [25] were the only authors to use autogenous bone from an extraoral donor part (iliac crest), thereby requiring patients to receive general anesthesia in a hospital setting. Although we only have results of an extraoral donor site from one study, the iliac crest presented less bone gain than did the grafts from intraoral areas used in the other four studies. Nevertheless, this fact did not preclude the possibility of implant installation.

Structurally, the pattern of bone remodeling in autogenous grafts is closely related to the cortical or medullary graft pattern $[5,28]$. Thus, when a cortical bone (mandibular ramus) is compared to a corticomedullary bone (iliac crest), greater potential for resorption is expected. Consequently, lower bone gain in the long term is expected in the bone with a greater amount of bone marrow tissue [28-30], which justified the histological data and the parameters measured by the studies selected in this review [24-27]. Therefore, autogenous grafting from extraoral areas is not necessary when grafting with this technique and in this region of bone atrophy. This explains why a majority of studies use intraoral bone grafts. Consequently, there is no difference between the complication rates, as the extraoral grafts are subject to greater morbidity and postoperative complications [10,31].

A limitation of the included studies is that data only on bone gain before implant installation were evaluated, even though vertical augmentation depends on several factors such as surgical methods, type of prostheses, bone graft, and systemic conditions. However, a sub-analysis based on these factors was not performed due to absence of specific data in the included studies.

Regarding the guided bone regeneration technique employed, there was similarity in the meta-analysis conducted for the parameter postoperative complications, regardless of the grafting or fixation/stabilization material used. However, the autogenous group showed a favorable trend toward displacement (as shown by the diamond) in the 


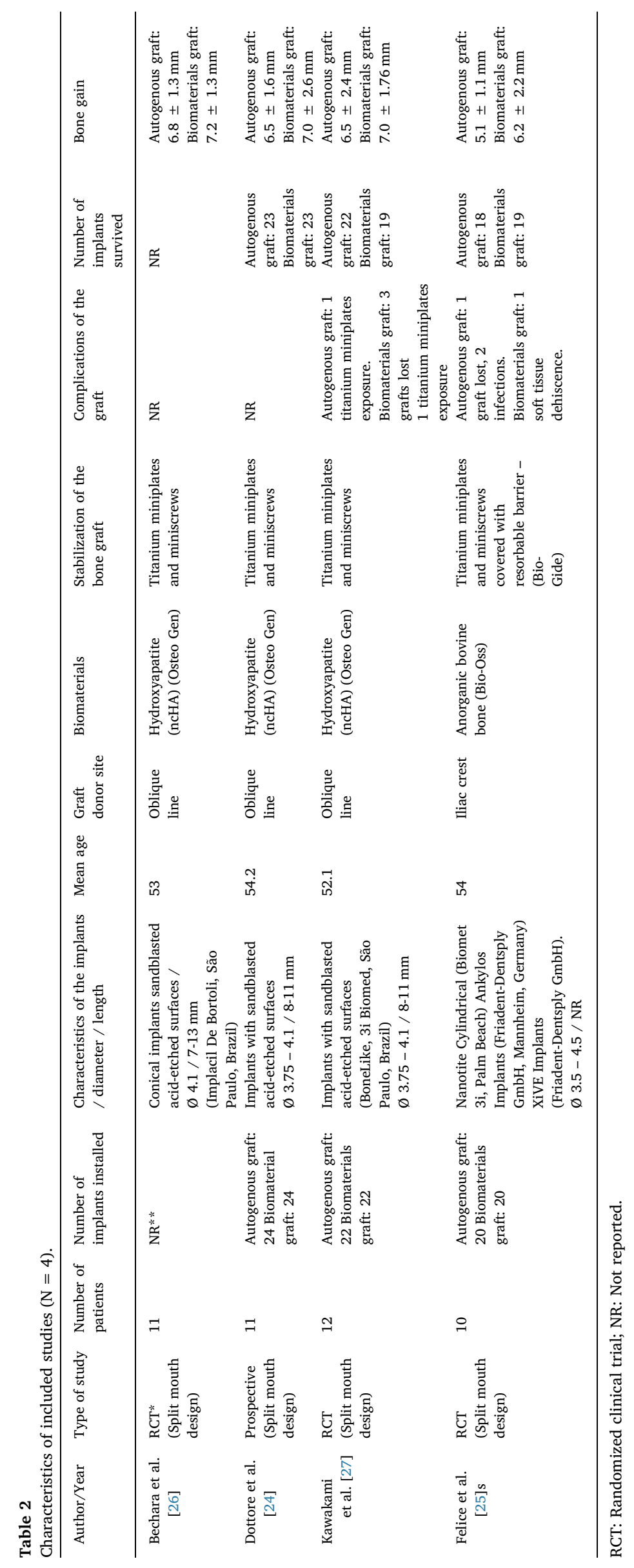




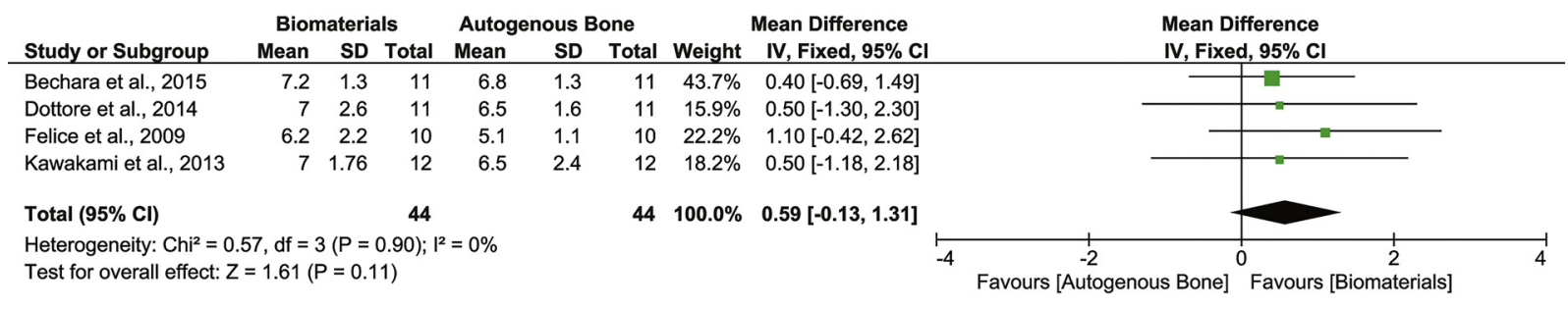

Fig. 2. Forest plot comparing bone substitutes with autogenous bone graft for bone gain.

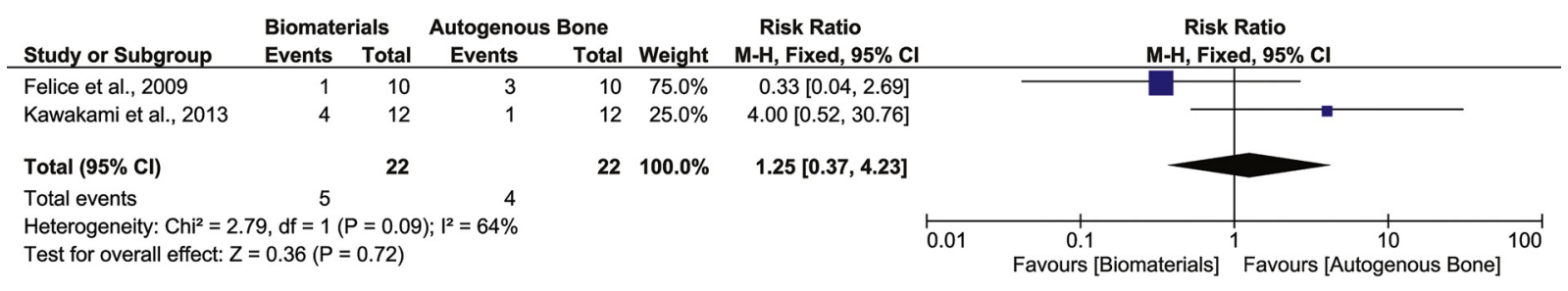

Fig. 3. Forest plot comparing bone substitutes with autogenous bone graft for complications rate.

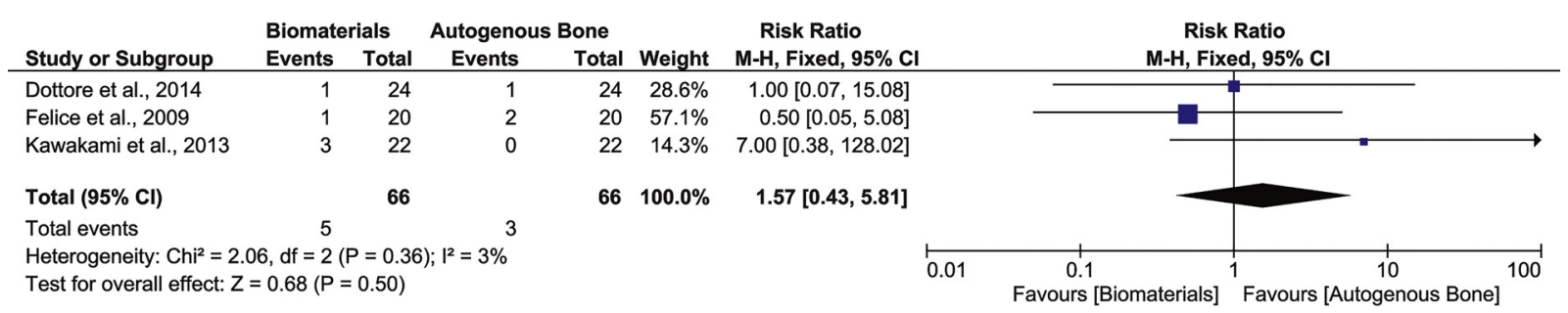

Fig. 4. Forest plot comparing bone substitutes with autogenous bone graft for implant survival rate.
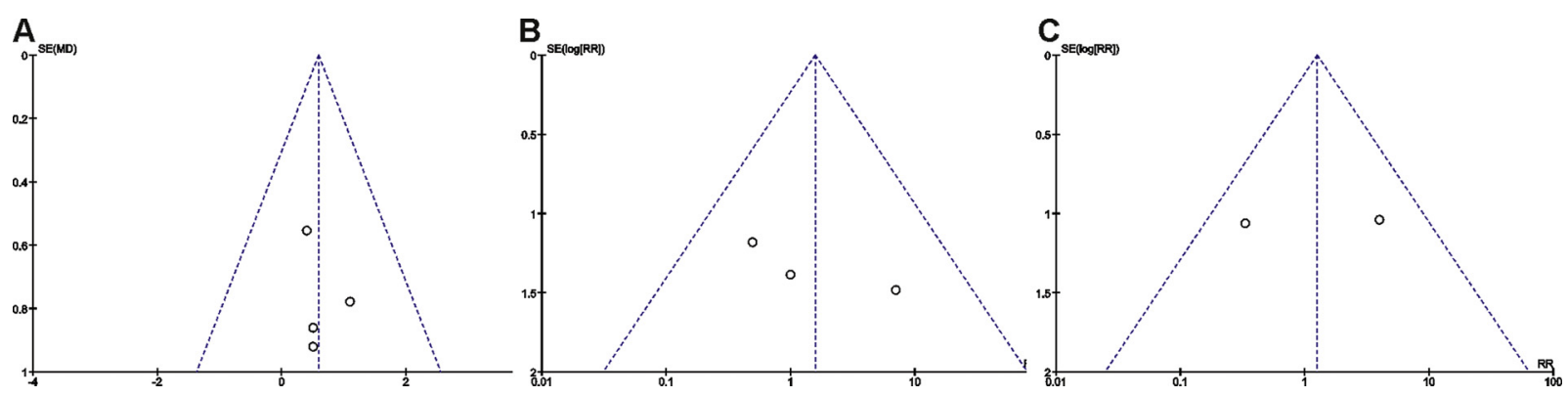

Fig. 5. Funnel plot of each outcome evaluated (A: Bone gain; B: Complication rate; C: Implant survival rate).

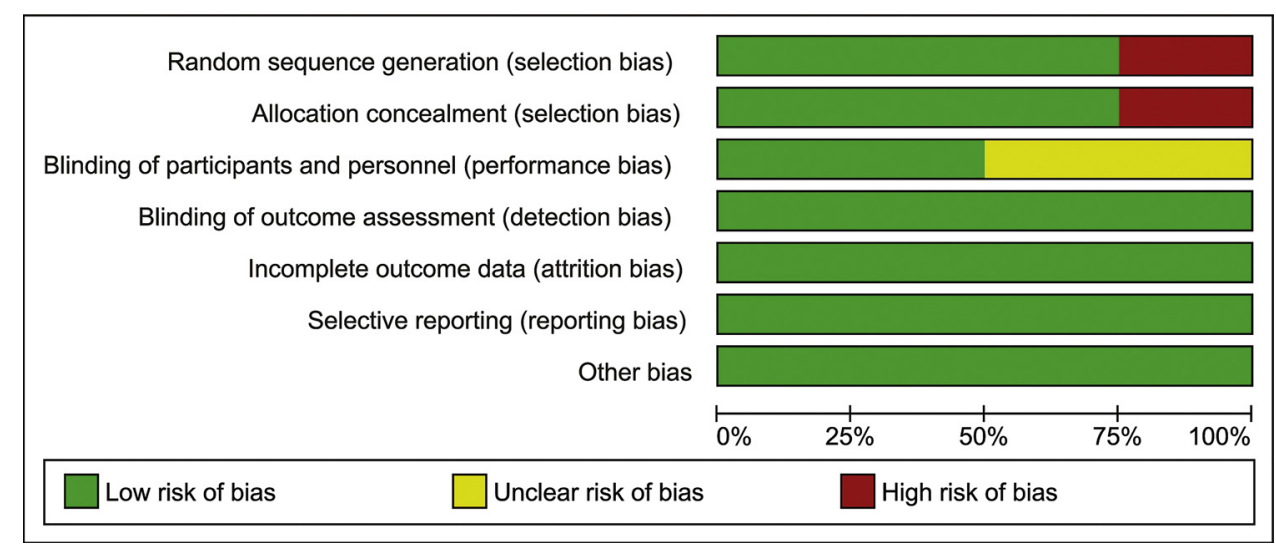

Fig. 6. Assessment of the risk of bias in the included studies based on the Cochrane Risk of Bias tool. 
forest-plot graph (Fig. 3). These data are probably justified by the greater potential for bone remodeling that is necessary for the integration of the autogenous grafts than for strictly osteoconductive biomaterials, which behave as a support framework for new bone formation. Although complications due to the use of titanium meshes were expected [32], only two cases with this complication were reported in included studies. Furthermore, the exposure of titanium miniplates did not affect the installation of implants.

The primary stability parameter of the implant as well as the survival rate of the implant during a follow-up period of 5-12 months was similar between the two types of grafts. Only Bechara et al. [26] reported the comparative values regarding the initial stability of the implants, presenting mean implant stability quotient values of 77.94 for the autogenous group and 74.69 for the biomaterial group. However, the other authors [24,27] generalized that the primary stability was satisfactory for all implants, independent of the graft type.

The complications reported by Dottore et al. [24] were restricted to a total of four implant losses due to failure in osseointegration, two cases of peri-implantitis, and one case of infection. A follow-up period of at least 2 months and a maximum of 6 months after the installation of the prosthesis was reported by the studies [25-27]. Among the studies with a follow-up period of 1-2 years, Dottore et al. [24] reported marginal bone loss of up to $1 \mathrm{~mm}$ for autogenous bone and $0.78 \mathrm{~mm}$ for biomaterials. Felice et al. [25] reported the loss of one implant in the biomaterial group; the other studies did not report data regarding these parameters.

In this systematic review, all biomaterials were considered for inclusion criteria independently of the biomaterial type. Three selected studies used hydroxyapatite (ncHA) and one used an organic bovine bone, which made it impossible to carry out a sub-analysis based on the type of biomaterial. However, to reduce the heterogeneity between selected studies, we only included articles with a direct comparison using the split-mouth design.

On the basis of these results, we recommend that future studies should outline the same methodology as that of previous studies and initially standardize the follow-up with the measurement of bone loss parameters and complication rates after implantation, as well as perform long-term follow-up of at least 5 years. This analysis is limited by the differences in the surgical techniques and fixation of grafts performed in the studies assessed. The follow-up periods after implantation were also insufficient. In addition, standardization of the type of connection and surface treatment of the implants is not possible.

Nevertheless, this systematic review and meta-analysis clarifies that the rates of vertical bone gain, primary implant stability, implant survival, and postoperative complications are very similar between autogenous bone grafts and biomaterials used for addressing posterior mandibular atrophy.

\section{Conclusion}

The current meta-analysis indicates that biomaterials or autogenous bone are indicated for the reconstruction of the posterior mandibular atrophic region, without lowering implant survival.

\section{Conflict of interest}

The authors declare no conflict of interest.

\section{References}

[1] F. Carini, S. Longoni, E. Amosso, J. Paleari, S. Carini, G. Porcaro, Bone augmentation with TiMesh. Autologous bone versus autologous bone and bone substitutes. A systematic review, Annali di Stomatologia 5 (2014) 27-36.

[2] R. Adell, U. Lekholm, B. Rockler, P.I. Branemark, A 15-year study of osseointegrated implants in the treatment of the edentulous jaw, Int. J. Oral Surg. 10 (1981) 387-416.

[3] A. Leonhardt, K. Grondahl, C. Bergstrom, U. Lekholm, Long-term follow-up of osseointegrated titanium implants using clinical, radiographic and microbiological parameters, Clin. Oral Implants Res. 13 (2002) 127-132.

[4] D. Buser, S. Ingimarsson, K. Dula, A. Lussi, H.P. Hirt, U.C. Belser, Long-term stability of osseointegrated implants in augmented bone: a 5-year prospective study in partially edentulous patients, Int. J. Periodont. Restor. Dent. 22 (2002) 109-117.

[5] M. Chiapasco, M. Zaniboni, M. Boisco, Augmentation procedures for the rehabilitation of deficient edentulous ridges with oral implants, Clin. Oral Implants Res. 2 (2006) 136-159.

[6] N. Donos, N. Mardas, V. Chadha, Clinical outcomes of implants following lateral bone augmentation: systematic assessment of available options (barrier membranes, bone grafts, split osteotomy), J. Clin. Periodontol. 35 (2008) 173-202.

[7] M. Clementini, A. Morlupi, L. Canullo, C. Agrestini, A. Barlattani, Success rate of dental implants inserted in horizontal and vertical guided bone regenerated areas: a systematic review, Int. J. Oral Maxillofac. Surg. 41 (2012) 847-852.

[8] M. Hallman, A. Thor, Bone substitutes and growth factors as an alternative/complement to autogenous bone for grafting in implant dentistry, Periodontology 47 (2008) 172-192 2000.

[9] E. Nkenke, F. Stelzle, Clinical outcomes of sinus floor augmentation for implant placement using autogenous bone or bone substitutes: a systematic review, Clin. Oral Implants Res. 20 (Suppl 4) (2009) 124-133.

[10] A. Barone, M. Ricci, F. Mangano, U. Covani, Morbidity associated with iliac crest harvesting in the treatment of maxillary and mandibular atrophies: a 10-year analysis, J. Oral Maxillofac. Surg. 69 (2011) 2298-2304.

[11] E. Nkenke, V. Weisbach, E. Winckler, P. Kessler, S. Schultze-Mosgau, J. Wiltfang, F.W. Neukam, Morbidity of harvesting of bone grafts from the iliac crest for preprosthetic augmentation procedures: a prospective study, Int. J. Oral Maxillofac. Surg. 33 (2004) 157-163.

[12] C.H. Hammerle, G.C. Chiantella, T. Karring, N.P. Lang, The effect of a deproteinized bovine bone mineral on bone regeneration around titanium dental implants, Clin. Oral Implants Res. 9 (1998) 151-162.

[13] M. Piattelli, G.A. Favero, A. Scarano, G. Orsini, A. Piattelli, Bone reactions to anorganic bovine bone (bio-oss) used in sinus augmentation procedures: a histologic long-term report of 20 cases in humans, Int. J. Oral Maxillofac. Implants 14 (1999) 835-840.

[14] P.A. Fugazzotto, GBR using bovine bone matrix and resorbable and nonresorbable membranes. Part 2: clinical results, Int. J. Periodont. Restor. Dent. 23 (2003) $599-605$.

[15] N.U. Zitzmann, P. Scharer, C.P. Marinello, P. Schupbach, T. Berglundh, Alveolar ridge augmentation with bio-oss: a histologic study in humans, Int. J. Periodont. Restor. Dent. 21 (2001) 288-295.

[16] L. Cordaro, D.S. Amade, M. Cordaro, Clinical results of alveolar ridge augmentation with mandibular block bone grafts in partially edentulous patients prior to implant placement, Clin. Oral Implants Res. 13 (2002) 103-111.

[17] A. Khojasteh, S.R. Motamedian, N. Sharifzadeh, H.H. Zadeh, The influence of initial alveolar ridge defect morphology on the outcome of implants in augmented atrophic posterior mandible: an exploratory retrospective study, Clin. Oral Implants Res. 28 (2016) 208-217.

[18] A. Restoy-Lozano, J.L. Dominguez-Mompell, P. Infante-Cossio, J. Lara-Chao, F. Espin-Galvez, V. Lopez-Pizarro, Reconstruction of mandibular vertical defects for dental implants with autogenous bone block grafts using a tunnel approach: clinical study of 50 cases, Int. J. Oral Maxillofac. Surg. 44 (2015) 1416-1422.

[19] G. Morad, A. Khojasteh, Cortical tenting technique versus onlay layered technique for vertical augmentation of atrophic posterior mandibles, Implant Dent. 22 (2013) 566-571.

[20] D. Moher, A. Liberati, J. Tetzlaff, D.G. Altman, Preferred reporting items for systematic reviews and meta-analyses: the PRISMA statement, Int. J. Surg. 8 (2010) 336-341.

[21] B. Al-Nawas, E. Schiegnitz, Augmentation procedures using bone substitute materials or autogenous bone - a systematic review and meta-analysis, Eur. J. Oral Implantol. (2014) S219-234.

[22] S. Annibali, I. Bignozzi, M.P. Cristalli, F. Graziani, G. La Monaca, A. Polimeni, Periimplant marginal bone level: a systematic review and meta-analysis of studies comparing platform switching versus conventionally restored implants, J. Clin. Periodontol. 39 (2012) 1097-1113.

[23] M.A. Atieh, H.M. Ibrahim, A.H. Atieh, Platform switching for marginal bone preservation around dental implants: a systematic review and meta-analysis, J. Periodontol. 81 (2010) 1350-1366.

[24] A.M. Dottore, P.Y. Kawakami, K. Bechara, J.A. Rodrigues, A. Cassoni, L.C. Figueiredo, A. Piattelli, J.A. Shibli, Stability of implants placed in augmented posterior mandible after alveolar osteotomy using resorbable nonceramic hydroxyapatite or intraoral autogenous bone: 12-month follow-up, Clin. Implant Dent Relat. Res. 16 (2014) 330-336.

[25] P. Felice, C. Marchetti, G. Iezzi, A. Piattelli, H. Worthington, G. Pellegrino, M. Esposito, Vertical ridge augmentation of the atrophic posterior mandible with interpositional bloc grafts: bone from the iliac crest vs. bovine anorganic bone. Clinical and histological results up to one year after loading from a randomized controlled clinical trial, Clin. Oral Implants Res. 20 (2009) 1386-1393.

[26] K. Bechara, A.M. Dottore, P.Y. Kawakami, S.A. Gehrke, P.G. Coelho, A. Piattelli, G. Iezzi, J.A. Shibli, A histological study of non-ceramic hydroxyapatite as a bone graft substitute material in the vertical bone augmentation of the posterior mandible using an interpositional inlay technique: a split mouth evaluation, Ann. Anat. 202 (2015) 1-7.

[27] P.Y. Kawakami, A.M. Dottore, K. Bechara, M. Feres, J.A. Shibli, Alveolar osteotomy associated with resorbable non-ceramic hydroxylapatite or intra-oral autogenous bone for height augmentation in posterior mandibular sites: a split-mouth prospective study, Clin. Oral Implants Res. 24 (2013) 1060-1064. 
[28] P.S. de Carvalho, L.W. Vasconcellos, J. Pi, Influence of bed preparation on the in corporation of autogenous bone grafts: a study in dogs, Int. J. Oral Maxillofac. Implants 15 (2000) 565-570.

[29] H. Burchardt, The biology of bone graft repair, Clin. Orthop. Relat. Res. 174 (1983) $28-42$.

[30] B.L. Hammack, W.F. Enneking, Comparative vascularization of autogenous and homogenous-bone transplants, J. Bone Jt. Surg. Am. 42A (1960) 811-817.
[31] C.E. Misch, M.W. Bidez, M. Sharawy, A bioengineered implant for a predetermined bone cellular response to loading forces. A literature review and case report, J Periodontol. 72 (2001) 1276-1286.

[32] M. Rasia-dal Polo, P.P. Poli, D. Rancitelli, M. Beretta, C. Maiorana, Alveolar ridge reconstruction with titanium meshes: a systematic review of the literature, Med. Oral Patol. Oral. Cir. Bucal. 19 (2014) e639-646. 